prehensive surgical textbook but is a comprehensive text in the practical aspects of surgery and should appeal to its readership. This is clearly aimed at the peri-membership trainee and, as such, is a valued addition to the subject, giving a slightly different aspect to other texts in this field. Each of the 31 chapters is well researched and written, covering the whole field of practical surgery including peri-operative care, laparoscopic surgery, day surgery, obesity, surgery of the elderly, etc. The last four chapters look at the common tropical diseases as they affect general surgical practice - hydatid, schistosomiasis, tuberculosis and typhoid. These diseases are becoming more common in Western medicine and their inclusion makes this book widely relevant.

There are a large number of authors, drawn from the UK and internationally. Each chapter, therefore, has a slightly different writing style. This actually adds to the text, making it very readable, and the editing has drawn the different chapters together - exemplified by the high-lighted key points throughout and the summary and question sections at the end of each chapter. There are excellent bibliographies throughout, which are concise and up to date. This is a well-written text suited for all peri-MRCS trainees and I highly recommend it.

\section{A Guide to Cancer Genetics in Clinical Practice}

SUE CLARK

\begin{tabular}{ll}
\hline EXTENT P/H & 238 pages, Paperback \\
PRICE/ISBN & $£ 30.00,9781903378540$ \\
PUBLISHER & tfm (Shrewsbury), 2009 \\
REVIEWERS & Diana Eccles and Andrew Douglas \\
STAR RATING & $* * *$
\end{tabular}

This concise, multi-authored textbook on cancer genetics sets out to introduce clinicians to common inherited cancer syndromes and allow identification of at-risk individuals and families. It is orientated towards a surgical audience. With edited textbooks there is a balance between individual author expression and a unifying style. This book favours individual approaches in general and reads like a series of essays. The absence of an index enhances this impression.

Every chapter has a 'key summary points' section and some have useful management algorithms. The introductory chapter on basic genetics is an elementary introduction at medical-student level, which contrasts with some much more complex genetic detail in later chapters (e.g. the NF1 chapter). The chapter on ethical and legal aspects with illustrative case histories is a useful one. While many chapters are informative, clear and balanced, such as the chapters on FAP and MEN syndromes, others seem to lack a wider context. For a largely surgical audience, the inclusion of NF1 and not NF2 is surprising. A chapter on inherited renal cancer might have been a useful addition and perhaps more relevant than a chapter on hereditary pancreatic cancer.

Some authors present justification or are clear about the lack of evidence for suggested surveillance strategies; others advocate intensive surveillance without any supporting evidence. For example, CT scanning and small-bowel series every year or so would expose a patient with Peutz-Jegher's syndrome to a considerable cumulative radiation dose without clear evidence of benefit. The presentation of risks and genetic testing is generally good but there are a few notable errors. For example, a $25 \%$ risk of pancreatic cancer in cystic fibrosis is very misleading (source not referenced). The competing mortalities for CF patients are such that pancreatic cancer mortality, although increased about 5-fold over the population risk, is actually negligible.

The final summary chapter looks more at the future rather than summarising the book's contents and, unfortunately, it mixes somatic and inherited genetic changes in a somewhat confusing way. This book contains a lot of solid and useful information about cancer genetics and as an introductory text for non-specialist clinicians it has much to offer. However, subsequent editions would certainly benefit from a more consistent style and an index. 